CIMSA 2008 - IEEE International Conference on

Computational Intelligence for Measurement Systems and Applications

Instabul, Turkey, 14-16 July 2008

\title{
Image Processing for Granulometry Analysis via Neural Networks
}

\author{
Stefano Ferrari, Vincenzo Piuri, and Fabio Scotti \\ Dipartimento di Tecnologie dell'Informazione \\ Università degli Studi di Milano \\ Via Bramante 65 \\ 26013 Crema, Italy \\ e-mail: fferrari, piuri, fscotti\}@dti.unimi.it
}

\begin{abstract}
The analysis of granulometry of substances is relevant in a great variety of the research and industrial applications as such as the pharmaceutical sector, the food sector, the basic materials production and in the concrete and wood panel industries. This analysis is important since many relevant properties of the materials can depend on the distribution of the particles sizes/shapes during the production. In this work we present an innovative method capable to estimate the particles size distribution in an image without the use of segmentation techniques by using neural networks. The paper contribution is twofold. The proposed method presents a set of techniques based on wavelet analysis and image processing techniques suitable to extract relevant features for the granulometry analysis. Then, the extracted set of features is used as input to neural networks in order to achieve the classification of each single pixel accordingly to the probability to belong to a specific class of particles size (a single band in the histogram of the distribution of the particles size). The produced outputs have been used to perform the estimation of the particle granulometry contained in the image. Results are encouraging and show the effectiveness of the proposed method.
\end{abstract}

Keywords - Granulometry analysis, neural networks, image processing, wavelet filtering.

\section{INTRODUCTION}

The analysis of granulometric properties of substances is relevant in a great variety of the research as well as in the industrial applications which can range from the pharmaceutical sector to the food sector, papermaking and coating, basic materials production and in many innovative applications. In particular, the analysis of the granulometry analysis of the materials can be very important since the properties and the characteristics of the final products are strongly correlated to the distribution of the shapes and sizes of the particles composing the mixtures and ingredients used in the productions [1]. In addition, the granulometry analysis can also enlighten problems occurred during the production operations (bias in the machines working point, wearing of the tools, wrong or poor quality in the basic materials, etc.) [2].

The distribution of the particles composing the basic material and mixtures of a product can greatly affect the final quality and properties. For example, in the wood panel production, a

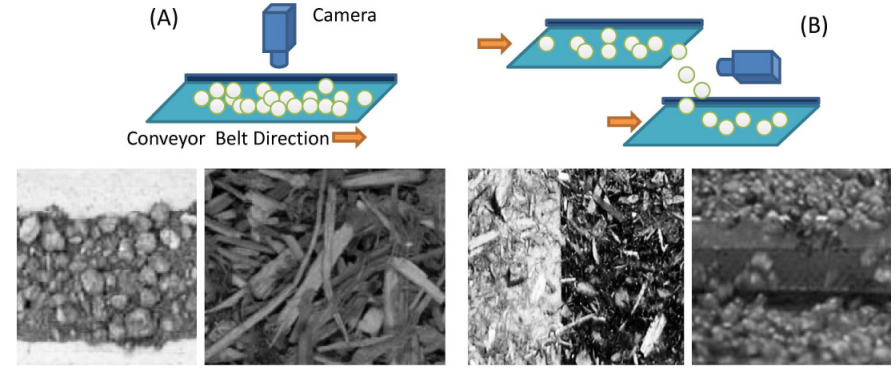

Fig. 1. Typical setups for vision-based granulometric analysis and four image examples. Upper subplots: (A) in-line scanning setup, by using a top view camera and (B) free fall setup. Bottom images, from left to right: oil sands on a moving conveyor belt, wood chips acquired with the setup A and B respectively, coffee seeds acquired with the setup B.

mixture of chopped wood particles are glued and pressed in order to obtain a suitable raw chip board. The proper value of the glue/wood ratio is strongly related to the size and shape of the particles composing the panel. This setup value affects the final mechanical properties of the panel, its cost and the environmental impact related to the presence of formaldehyde and other pollutants contained in the glue inside the panel [3].

Up to now, the most common method to estimate the granulometry consists of a mechanical division of the particles by using vibrating sifters, followed by a weighting operation of the obtained partitions. This estimation suffers of many drawbacks: the analysis is made by human experts on a single sample of the production, it is not rapid and its accuracy is hardly standardizable due to the operator's capabilities and the correct application of the measurement procedures.

Some innovative systems exploit vision-based approaches in order to estimates the distributions of the objects present in a region of interest of the acquired image of the material. In this case the procedures can occur without contact and the procedure can be fully automated. In this case, the systems can work on single amount of the product extracted from the production line (spin-off approaches) or directly on the production line (in-line approaches). Figure 1 show two different setups for in-line approaches.

Setups which exploit a top-view of the particles (setup (A) in Fig. 1) are very simple and effective but the obtained images 
are far to be ideal since most of the time the particle are superimposed. Free-fall setups (setup (B) in Fig. 1) allow for a better separation of the particles in the region of interest of the camera, but out-of-focus problems can occur and very short exposure time are required in order to acquire proper still images combined to an effective illumination system.

The paper shows that it is effectively possible to estimate the particles size distribution in an image by using a global approach based on neural networks without the use of segmentation techniques. In particular, the proposed method presents a set of techniques suitable to extract features from the input image which are effective in the granulometry analysis. Then, the extracted features have been used to train inductive classifiers (such as neural networks) to classify each single image pixel in a set of size classes. Results are interesting and they show that satisfactory accuracy can be obtained. The structure is the following. In the next section the state of the art is presented, then the proposed method is presented. In the last section it is presented the creation of a synthetic dataset, and the experimental results of the proposed method are presented and discussed.

\section{PREVIOUS WORK}

Vision-based systems estimate the distribution of the objects present in a region of interest of the acquired image by a two-steps process: first a segmentation operation is processed in order to locate each object in the image; in the second step, the evaluation of the properties of the objects identified during the segmentation is performed. The more objects have been correctly measured, the more confident will be the estimation of the particles size distribution.

Similar approaches have been used in biological applications in order to test the health of the human tissue/cells based on successive structural openings of the segmented image [4] [5] [6] [7], and in [8] is discussed the case of non-organic particles. The segmentation phase is typically present in the classical image-based granulometry analysis, and it represents the most important contribution of errors in the estimation when the setup is not controlled (e.g., when objects/particles are superimposed, and/or the foreground has a non correct illumination). Moreover, a proper segmentation of a scene that is complex and densely populated of superimposed elements is typically a quite computationally intensive task. To overcome this problem, in [9] and in [10] global approaches have been proposed without using segmentation applying the morphological pattern spectrum and the Fourier spectrum.

More complex setups than a single camera can be also be adopted. For example, in [11] a 3D laser scanner has been used to detect the surface of wood chips on a conveyor belt on. Unfortunately, the paper does not compare the results of the proposed system with respect to the results based on a single camera setup, hence is not possible to determine if the aug- mented complexity of the setup is balanced with a higher accuracy of the system.

In this paper we propose an innovative image-based granulometric analysis technique which avoid the use of the segmentation and its related problems above described.

\section{THE PROPOSED METHOD}

The granulometry problem can be formalized as follow: given an image $I(x, y)$, find out the distribution $p(r)=$ $\{P($ an object depicted in $I$ has size $r)\}, r \in \mathbb{R}^{+}$. Usually, for practical application, the range of the objects' size can be partitioned in few intervals, defining a set of size classes $\left\{C_{i} \mid r_{i}<\right.$ $\left.r \leq r_{i+1}\right\}$ (for a set of suitable increasing values $\left\{r_{i}, i=\right.$ $\left.\left.0, \ldots, M \mid r_{i}<r_{i+1}, r_{0}=0\right\}\right)$, moving the focus from $p(r)$ to $p\left(C_{i}\right)=\left\{P\left(\right.\right.$ an object depicted in $I$ belongs to $\left.\left.C_{i}\right)\right\}$.

Segmentation-based granulometry techniques identify an ensemble of objects $\left\{O_{k}\right\}$ in $I(x, y)$ and obtain $p\left(C_{i}\right)$ by measuring the size of each object in $\left\{O_{k}\right\}$. Hence, the pixels of the image are partitioned in regions (a region for each object) and the size of the object is then measured over the pixels that form the object itself (e.g., the distance of the most distant pair of pixels).

We propose a method that works in another way: instead of partitioning the image in objects, we estimate, for each pixel $(x, y)$ the size $R(x, y)$ (or the class $C(x, y)$ ) of the the object the pixel belongs to. Then, we obtain $p(r)$ (or $p\left(C_{i}\right)$ ) as a statistics of $R(x, y)$ (or $C(x, y)$ ). Thus, we obtain the distribution of an object's property (e.g., the object's size) from the distribution of the pixels' property (e.g., the object's size the pixel belongs to). It should be noted that from the knowledge of $R(x, y)$ it is possible to estimate $p(R)=\{P($ a pixel of $I$ belongs to $R$-sized object $)\}$, but some processing is needed to obtain $p(r)$, as the number of pixels taken up for a given object does not only depend on the object's size but also rely on its shape. For instance, for a circular shaped object, the number of pixels that belong to the object will be proportional to the square of the object's radius, while for a needle shaped object, the number of pixels will be proportional to the object's length. Hence, some mild a priori knowledge on the objects' shape allows to avoid the need of segmenting the image and allow to obtain the relation between the object's shape and its area that can be used for transforming $p(R)$ into $p(r): p(r)=T(p(R))$, or $p(C)$ into $p\left(C_{i}\right): p\left(C_{i}\right)=T(p(C))$. We will refer to the transformation $T$ as shape-based correction of the distribution.

If, for each pixel $(x, y)$, the function $R(x, y)$ was known, the granulometry problem would be trivial. However, it is not unreal suppose a partial knowledge of $R(x, y)$ (or $C(x, y)$ ) (e.g., from manual measurement). This allows to reframe the problem of estimating the distribution of the size of the objects of a scene as a problem of function approximation (classification) from examples. In the following we will consider only 


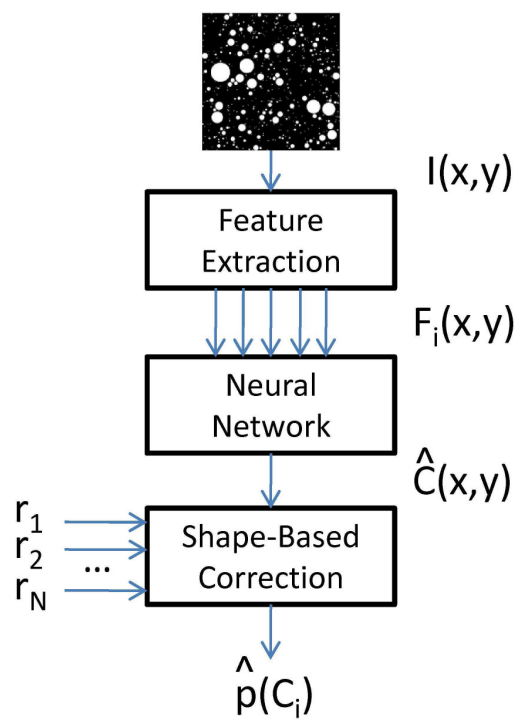

Fig. 2. Structure of the modules composing the proposed approach.

the classification case, as it is more interesting for practical applications.

The proposed method (Fig. 2) is based on the observation that the persistence of an object in an image under the operation of low-band filtering is proportional to the size of the object. Hence, a multi-scale representation of the image can be used to estimate the size of the objects depicted in the image.

The scale-space representation [12] of a signal is a collection of blurred copies of the original signal, usually obtained convolving the signal with a Gaussian filter. As the cutfrequency of the filter increases, details at small scale fades out.

Given the image $I(x, y)$, the set of $k$ features is given by:

$$
F_{i}(x, y)=I(x, y) * G\left(x, y ; \sigma_{i}\right)
$$

where $G(\cdot, \cdot ; \sigma)$ is a Gaussian kernel whose width is $\sigma$ (the larger is $\sigma$, the more blurred is the filtered image). The set $\left\{\sigma_{1}, \ldots, \sigma_{k}\right\}$ determines the scales at which the image $I$ is analyzed (Fig. 4).

We propose to use a neural network to approximate the function $f$ such that:

$$
C(x, y)=f\left(F_{1}(x, y), \ldots, F_{k}(x, y)\right)
$$

Summarizing, the granulometer presented in this paper takes a bitmap as input and produce an histogram of the class of the objects depicted in the input bitmap, by using the procedure represented in Fig. 2. The input bitmap is processed for obtaining its scale-space representation, then the corresponding pixels in each feature bitmap is fed to the neural classifier which predicts the class the pixel belongs to. Finally, from the pixels' classes histogram, the objects' classes histogram is obtained through shape-based correction.

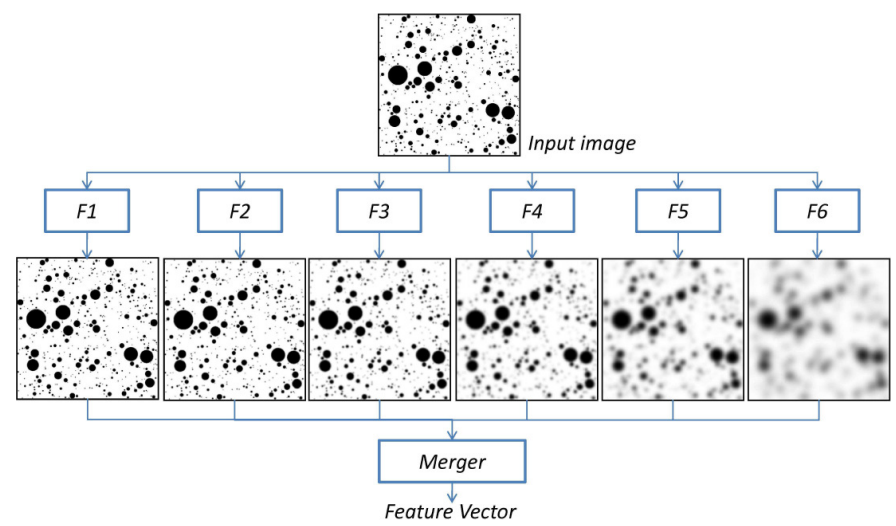

Fig. 3. Creation of the feature vectors. The original image is filtered by a bank of filter $F 1-F 6$, and the processed images are merged in order to produce the feature vector used in input to the neural networks. Each pixel of the original image is transformed in a 6 real-valued vector.
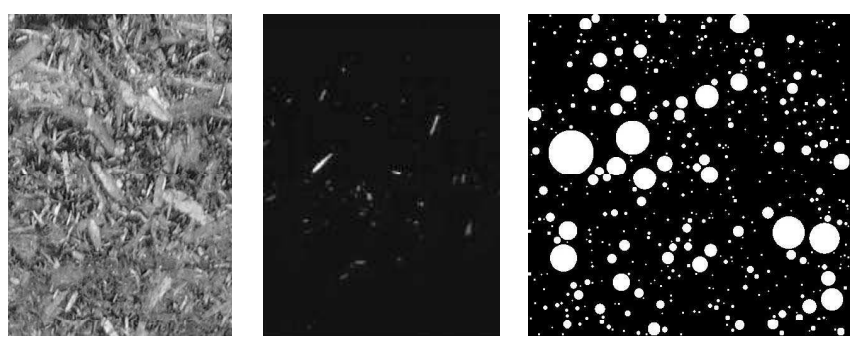

Fig. 4. Comparison of the real and synthetic images. From the left: real dense and sparse free-fall distributions; an example of a synthetic image.

\section{EXPERIMENTAL RESULTS}

In order to test the effectiveness of the proposed approach, we generate a set of 12 bitmaps populated of black blobs on white background (such as the third image in Fig. 4). The bitmaps' size is $512 \times 512$ pixels, while the radius of the blobs ranged from 1 to 50 pixels, partitioned in six classes, as in Table I. Moreover, the class $C_{0}$ has been added to describe the background (i.e., the class to which belong to the pixel that do not belong to any blobs).

The classes distribution, $p\left(C_{i}\right)$ is reported in Table I. It has been evaluated on the entire set of bitmaps because the distribution of the classes may change from one bitmap to another.

For each bitmap, $I$, a set of seven features have been computed (Fig. 3): the first feature, $F_{0}$ has been defined as the bitmap $I$ itself, while the other features have been computed as in (1), by using a set of $\sigma$ defined as the mean blob's size of each class $(\{1.5,3.0,6.0,11.5,21.0,38.5\})$.

For approximating the class function, $C(x, y)$, in (2), we used a RBF network trained with hybrid learning [13] (NeuralGas [14] was used to distribute the centers of the neurons). The RBF network, composed by 50 Gaussian units, realizes a $\mathbb{R}^{k} \rightarrow \mathbb{R}^{M}$ function, where $k$ is the number of features, and $M$ is the number of the classes. For the setup we experimented, both $k$ and $M$ are equal to 7 . The $M$-valued RBF network 
TABLE I

CLASSES OF BLOBS' SIZE (IN PIXELS) AND THEIR TRUE AND PREDICTED DISTRIBUTIONS.

\begin{tabular}{ccc|cccccc|cc|} 
& & & \multicolumn{9}{|c|}{$\hat{p}_{100}\left(C_{i}\right)$} & \multicolumn{2}{c|}{$\hat{p}_{1000}\left(C_{i}\right)$} \\
$C_{i}$ & size range & $p\left(C_{i}\right)$ & RBF & LIN & QUAD & $1-\mathrm{NN}$ & 3-NN & 31-NN & RBF & $1-\mathrm{NN}$ \\
\hline$C_{0}$ & 0 & & & & & & & & & \\
$C_{1}$ & {$[1,2]$} & 0.557 & 0.552 & 0.601 & 0.826 & 0.607 & 0.609 & 0.611 & 0.561 & 0.545 \\
$C_{2}$ & $(2,4]$ & 0.261 & 0.261 & 0.318 & 0.168 & 0.240 & 0.237 & 0.222 & 0.259 & 0.302 \\
$C_{3}$ & $(4,8]$ & 0.125 & 0.123 & 0.0424 & 0.00528 & 0.0924 & 0.0941 & 0.113 & 0.126 & 0.103 \\
$C_{4}$ & $(8,15]$ & 0.0431 & 0.0490 & 0.0308 & 0.000465 & 0.0472 & 0.0461 & 0.0382 & 0.0396 & 0.0367 \\
$C_{5}$ & $(15,27]$ & 0.0110 & 0.0138 & 0.00699 & $3.8210^{-6}$ & 0.0107 & 0.0107 & 0.0118 & 0.0117 & 0.00992 \\
$C_{6}$ & $(27,50]$ & 0.00256 & 0.00203 & 0.00130 & $1.8310^{-5}$ & 0.00352 & 0.00371 & 0.00398 & 0.00242 & 0.00290
\end{tabular}

TABLE II

MEAN CONFUSION MATRIX OF THE RBF CLASSIFIER (100 EXAMPLES PER CLASS).

\begin{tabular}{c|ccccccc} 
& $C_{0}$ & $C_{1}$ & $C_{2}$ & $C_{3}$ & $C_{4}$ & $C_{5}$ & $C_{6}$ \\
\hline$\hat{C}_{0}$ & $2.2610^{5}$ & 0 & 0 & 0 & 0 & 0 & 0 \\
$\hat{C}_{1}$ & 0.45 & 1790 & 128 & 0 & 0 & 0 & 0 \\
$\hat{C}_{2}$ & 83.9 & 14.2 & 3110 & 405 & 0.273 & 0.364 & 0 \\
$\hat{C}_{3}$ & 326 & 1.55 & 110 & 5580 & 789 & 0.545 & 0 \\
$\hat{C}_{4}$ & 2790 & 1.64 & 14.7 & 378 & 6497 & 290 & 9.27 \\
$\hat{C}_{5}$ & 789 & 0.364 & 7.09 & 66.0 & 763 & 6420 & 1330 \\
$\hat{C}_{6}$ & 1.00 & 0 & 5.36 & 74.0 & 157 & 274 & 4130
\end{tabular}

TABLE III

MEAN CONFUSION MATRIX OF THE 1-NN CLASSIFIER (100 EXAMPLES PER CLASS).

\begin{tabular}{c|ccccccc} 
& $C_{0}$ & $C_{1}$ & $C_{2}$ & $C_{3}$ & $C_{4}$ & $C_{5}$ & $C_{6}$ \\
\hline$\hat{C}_{0}$ & $2.2910^{5}$ & 0 & 0 & 0 & 0 & 0 & 0 \\
$\hat{C}_{1}$ & 0 & 1660 & 269 & 0 & 0 & 0 & 0 \\
$\hat{C}_{2}$ & 0 & 117 & 2640 & 298 & 0 & 0 & 0 \\
$\hat{C}_{3}$ & 0 & 4.18 & 317 & 4270 & 116 & 0 & 0 \\
$\hat{C}_{4}$ & 47.4 & 18.2 & 137 & 1780 & 6500 & 337 & 2.45 \\
$\hat{C}_{5}$ & 6.64 & 0.182 & 137 & 88.5 & 1410 & 5090 & 65 \\
$\hat{C}_{6}$ & 171 & 2.18 & 6.36 & 65.7 & 177.8 & 1560 & 5400
\end{tabular}

output is then used to predict the class the input pixel belong to by choosing the element of the RBF output that carries the greater value.

One bitmap has been used for obtaining the training data for the neural network, while the remaining 11 bitmaps have been used as test set of the granulometer. In particular, 100 examples for each class have been sampled for composing the training set.

The results are summarized in Table I, where the distribution obtained from the predicted class function, $\hat{C}(x, y)$, is compared with that obtained from the true class function, $C(x, y)$.

An accuracy comparison has been performed by comparing the confusion matrix of different classical classifiers. In our test we used a linear classifier (LIN), a quadratic classifier (QUAD) and three different $K$-NN with $K=1,3,31$ as ref-

\section{TABLE IV}

MEAN CONFUSION MATRIX OF THE 1-NN CLASSIFIER (1000 EXAMPLE PER CLASS).

\begin{tabular}{c|ccccccc} 
& $C_{0}$ & $C_{1}$ & $C_{2}$ & $C_{3}$ & $C_{4}$ & $C_{5}$ & $C_{6}$ \\
\hline$\hat{C}_{0}$ & $2.3010^{5}$ & 0 & 0 & 0 & 0 & 0 & 0 \\
$\hat{C}_{1}$ & 0 & 1780 & 87.2 & 2.36 & 0.272 & 0 & 0 \\
$\hat{C}_{2}$ & 0 & 20.3 & 3200 & 862 & 61.9 & 5.36 & 0 \\
$\hat{C}_{3}$ & 0 & 0.828 & 70.0 & 4750 & 845 & 0.363 & 0 \\
$\hat{C}_{4}$ & 0 & 0 & 12.4 & 752 & 6200 & 448 & 0 \\
$\hat{C}_{5}$ & 0 & 0 & 1.73 & 70.2 & 954 & 5510 & 144 \\
$\hat{C}_{6}$ & 0 & 0 & 3.45 & 59.7 & 150 & 1030 & 5320
\end{tabular}

erences. As overall figure of merit, we propose the weighted mean error on the predicted distribution, $\epsilon$, computed as follow:

$$
\epsilon=\sum_{i=1}^{M}\left|p\left(C_{i}\right)-\hat{p}\left(C_{i}\right)\right|
$$

The RBF classifier achieved $\epsilon=2.4810^{-3}$, while the more accurate reference classifier has resulted the $1-\mathrm{NN}$, which achieved $\epsilon=1.8210^{-2}$. In detail, the functioning of the classifiers can be described by the confusion matrix, in which the cell $(i, j)$ reports the count of the pixels belonging to the class $C_{j}$ that the classifier assign to the predicted class $\hat{C}_{i}$. In Tables III and III the confusion matrices (averaged over the eleven test datasets) of the RBF and 1-NN classifiers are provided. It is evident that when the classifier makes a error, the predicted class is mainly one of those closer to the true class (the confusion matrix is close to be diagonal). Although the the number of misclassification errors is relevant, they tend to compensate each other, resulting in an accurate estimation of the classes distribution.

In order to test the neural model with a proper ensemble of reference classifiers, we limited the number of training examples to 100 per class, do to the computational resources required by the $K$-NN classifier with high value of $K$.

However, increasing the number of training examples does not provide a relevant enhancement in accuracy. For example, using 1000 training examples for class, the RBF classifier achieved $\epsilon=1.7510^{-3}$, while the $1-\mathrm{NN}$ classifier achieved 
TABLE V

MEAN CONFUSION MATRIX OF THE RBF CLASSIFIER (1000 EXAMPLES PER CLASS).

\begin{tabular}{c|ccccccc} 
& $C_{0}$ & $C_{1}$ & $C_{2}$ & $C_{3}$ & $C_{4}$ & $C_{5}$ & $C_{6}$ \\
\hline$\hat{C}_{0}$ & 229000 & 0 & 0 & 0 & 0 & 0 & 0 \\
$\hat{C}_{1}$ & 5.18 & 1790 & 107 & 0 & 0 & 0 & 0 \\
$\hat{C}_{2}$ & 153 & 7.18 & 3110 & 248 & 0.272 & 0 & 0 \\
$\hat{C}_{3}$ & 27.3 & 0.636 & 130 & 5800 & 863 & 11.3 & 2.09 \\
$\hat{C}_{4}$ & 807 & 0.454 & 17.6 & 316 & 6400 & 350 & 5.27 \\
$\hat{C}_{5}$ & 17.8 & 0.181 & 4.64 & 54.7 & 746 & 6190 & 767 \\
$\hat{C}_{6}$ & 0.0909 & 0.272 & 4.73 & 79.3 & 192 & 435 & 4690
\end{tabular}

$\epsilon=1.4010^{-2}\left(\hat{p}_{1000}\right.$ in Table I $)$.

\section{CONCLUSIONS}

The paper presented an innovative image-based method performing the granulometric analysis via neural networks. In particular, the proposed method does not apply any segmentation techniques, avoiding many drawbacks related to this task. Moreover, it does not require any particular foreground/background illumination and positioning of the particle, which are common in other techniques proposed in the literature. Preliminary results on images with particles superimposed or attached each others are promising and suggest that the method could be applied in real cases.

The proposed method presents a set of techniques based on scale-space analysis and image processing techniques suitable to extract relevant features for the granulometry analysis. Then, the extracted set of features is used as input of a neural network-based classifier in order to achieve classification of each single pixel accordingly to the probability to belong to a specific class of particle sizes and a direct estimation of the local particle size of the pixel. The accuracy achieved on the synthetic test image dataset is in the order of few fraction of percents. Results are encouraging and the future work will study the application of the proposed method to datasets of real images and new method for extracting features.

\section{REFERENCES}

[1] G. Broderick, E. Cacchione, and Y. Heroux, "The importance of distribution statistics in the characterization of chip quality," Tappi journal, vol. 81, pp. 131-142, Aug. 1998.

[2] F. Ding, M. Benaoudia, P. Bdard, R. Lanouette, C. Lejeune, and P. Gagn, "Wood chip physical quality definition and measurement," Pulp and Paper Laboratory, Centre de recherche industrielle du Qubec (CRIQ), Quebec, Canada, Tech. Rep., 2005.

[3] P. Camorani, M. Badiali, D. Francomacaro, and M. Gamassi, "A classification method for wood types using fluorescence spectra," in IEEE International Instrumentation and Measurement Technology Conference, May 2008.

[4] V. Zapatera, L. Martnez-Costab, and G. Ayalaa, "A granulometric analysis of specular microscopy images of human corneal endothelia," Computer Vision and Image Understanding, vol. 97, no. 3, pp. 297-314, Mar. 2005.
[5] M. Colome-Serra, D. Seron, F. Moreso, M. Carrera, J. Grino, and J. Serra, "Image analysis: Utility of grey level granulometry to measure renal interstitial chronic damage," IEEE Engineering in Medicine and Biology Society, vol. 14, pp. 1934-1935, 1992.

[6] C. D. Ruberto, A. Dempster, S. Khan, and B. Jarra, "Automatic thresholding of infected blood images using granulometry and regional extrema," in International Conference on Pattern Recognition (ICPR'00), vol. 3, 2000, p. 3445 .

[7] N. Theera-Umpona, E. Doughertyb, and P. Gader, "Non-homothetic granulometric mixing theory with application to blood cell counting," Pattern Recognition, vol. 34, no. 12, pp. 2547-2560, Dec. 2001.

[8] F. Dornaika and H. Zhang, "Granulometry using mathematical morphology and motion," in IAPR Workshop on Machine Vision Applications, 2000, pp. 51-54.

[9] A. Zadorozny, H. Zhang, and M. Jagersand, "Granulometry using image transformation techniques," in International Conference on Vision Interface, 2002, pp. 433-438.

[10] A. Ledda, J. Quintelier, P. Samyn, P. D. Baets, and W. Philips, "Quantitative image analysis with mathematical morphology," in IAPR Workshop on Machine Vision Applications, 2000, pp. 51-54.

[11] M. Lpez, J. Matas, J. Viln, and J. Taboada, "Functional pattern recognition of $3 \mathrm{~d}$ laser scanned images of wood-pulp chips," Pattern Recognition and Image Analysis, vol. 4477, no. 8, pp. 298-305, Aug. 2007.

[12] A. Witkin, "Scale-space filtering," in Proc. 8th Int. Joint Conf. Art. Intell., 1983, pp. 1019-1022.

[13] J. Moody and C. J. Darken, "Fast learning in networks of locally-tuned processing units," Neural Computation, vol. 1, no. 2, pp. 281-294, 1989.

[14] T. Martinetz, S. Berkovich, and K. Schulten, "Neural-gas" network for vector quantization and its application to time-series prediction," IEEE Trans. on Neural Networks, vol. 4, no. 4, pp. 558-568, 1993. 